\title{
Dielectric response of disordered ferroelectrics with embedded charged clusters
}

\author{
A.N. Morozovska ${ }^{1}$, E.A. Eliseev ${ }^{2}$, V.V. Obukhovsky ${ }^{1}$ \\ ${ }^{1}$ Taras Shevchenko Kiev University, Radiophysical Department, 64 Volodymyrs'ka str., 01033 Kyiv, Ukraine \\ E-mail:morozo@mail.i.com.ua,obukhovsky@univ.kiev.ua. \\ ${ }^{2}$ Institute for Materials Science, NAS of Ukraine, 3 Krjijanovskogo, 03142 Kyiv, Ukraine
}

\begin{abstract}
The model of static charged clusters, which adequately describes the dielectric hysteresis and permittivity of disordered ferroelectrics with non-isovalent impurities, has been proposed. The main result of paper is modified Landau-Ginzburg-Devonshire equation can be applied to the bulk ferroelectric materials with improper conductivity and movable charge fluctuations near the impurity centers.
\end{abstract}

Keywords: disordered ferroelectrics, diffuse phase transition, charged defects.

Paper received 11.04.03; accepted for publication 16.06.03.

\section{Introduction}

Disordered ferroelectrics (ferroelectric ceramics, solid solutions of ferroelectric and non-ferroelectric components, composites, ferroelectrics doped with non-isovalent impurities, relaxors, etc.) are the materials with diffuse phase transition and sometimes the hysteresis loops changing caused by chemical composition fluctuations [1]. These peculiarities are the main difference between disordered ferroelectrics (DF) and ordered polar materials.

For example, the polarization in relaxors manifests the pronounce relaxation behavior, i.e. the temperature of dielectric response maximum depends on the frequency of applied electric field: this maximum shifts to the higher temperatures under the applied field frequency increasing in accordance with Vogel-Fulcheris law. But the dielectric permittivity value is more than $10^{3}$, which is common for ordered ferroelectrics. Also the polarization behavior is non-ergodic in relaxors.

The great variety of models has been proposed for the explanation of DF physical properties. The first model taking into account relaxors heterogeneous chemical composition has been introduced by Smolensky et al. [2] In this model the disorder in the system of B-site cations (for the composition of $\mathrm{A}\left(\mathrm{B}^{\prime} \mathrm{B}^{\prime \prime}\right) \mathrm{O}_{3}$ type with the perovskite structure) leads to the local phase transition temperature distribution, as a sequence phase transition becomes diffuse. In the ref. [3] the model of the hetero-phase fluctuations being the generalization of the previous model is proposed. The existence of microscopic regions (Känzig regions) with the gaussian distribution of chemical composition is the main assumption of this model. This is shown to lead to the broadening of the dielectric and electromechanical response of the system.

In the models proposed in refs. [4-8] relaxor ferroelectrics are considered as superparaelectrics, the phase transition diffusing is the result of the system mesoscopic nonhomogeneity (distribution of the polar regions sizes and the phase transition temperatures). Dielectric relaxation appears in this model due to the thermal activation of the polarization reorientation between the equivalent directions. In the ref. [6] DF is considered as an ideal superparaelectrics, i.e. an ensemble of independent polarized regions, each of them behaves as the ferroelectric of Landau-Ginzburg-Devonshire (LGD) type. Relaxor-like behaviour of the dielectric response is obtained in the framework of the models taking into account the distribution of the polar region sizes. As the model of relaxors with one transition temperature and the polar region size distribution these models correctly describe (numerically, not analytically) the shift of the temperature of dielectric response maximum with the frequency of external field.

In ref. [8] the mean field theory has been used for the description of the diffuse phase transition in the ferroelectrics with mesoscopic non-homogeneity. DF is treated as a multitude of polar nano-sized clusters in non-polar matrix. Clusters are described in the framework of the non-homogeneous phenomenological LGD theory, depolarization field, which can exist in the clusters, is neglected. 


\section{A.N. Morozovska et al.: Dielectric response of disordered ferroelectrics with ...}

In ref. [9], [10] the model of relaxors' dielectric properties based on the existence of polar clusters distributed in highly polarized lattice has been proposed. The obtained numerical results have been compared with experimental data. The random field theory for relaxors is presented in ref. [11]. The significance of consideration of charge carriers' localization at donor levels has been demonstrated in ref. [12].

Despite all the variety of existing models, none of them could give both: the simple qualitative explanation and analytical description of polarization switching phenomena and dielectric anomalies in DF. The main goal of our paper is to propose the rather general model of immovable charged clusters, which describes adequately the aforementioned DF properties and admits relatively simple analytical calculations. Taking into account that all the variety of DF chemical properties do not effect significantly on the qualitative behavior of their electrophysical properties, we try to involve the minimum number of hypothesis in our model. First of all we have not use the detailed description of the chemical nature, concentration and sizes' distribution of randomly situated immovable charged defects, which are the sources of movable charge carriers, inner electric field and induction fluctuations. That is why the main result of paper is modified LGD-equation (called analogical with modified Landau-Devonshire approach, evolved in Schmidt's papers [13]; see also comments in ref. [14]) can be applied to the bulk ferroelectric materials with improper conductivity and movable charge fluctuations near the impurity centers.

\section{The model of static charged clusters}

The proposed model admits the continuous transformation from the ordered LGD ferroelectric to DF and then to the completely disordered material under increasing the concentration of charged clusters.

If the hypothetical "pure", i.e. free of defects, sample (matrix) can be regarded as uniaxial ferroelectric, let us suppose that uniform external field $E_{0}(t)$ is applied along $z$-axis. If the matrix is perovskite, we assume that among three possible directions, the spontaneous polarization direction coincides with the external field one applied along one of the polar axes. This assumption is based on the following experimental fact [4]. Under cooling and further heating in zero-field (i.e. $E_{0}(t) \equiv 0$ the perovskite structure remains cubic or pseudo-cubic. Under cooling in the external field and further heating in zero-field the system behaves as ordered "uniaxial" ferroelectric, besides the spontaneous polarization direction is determined by the field one.

The static, i.e. immovable, defects (e.g. non-isovalent impurity) with fluctuating concentration are embedded to the matrix. We assume that even under the absence of proper conductivity impurity centers or clusters charge with charge density $\rho_{s}$ and so cause the rather high improper conductivity. That is why fluctuations $\delta \rho_{s}$ undoubtedly are the source of movable space-charge fluc- tuations $\delta \rho$ inner field $\delta E$ and induction $\delta D$ ones. The sample as a whole is electro-neutral. Evidently the local symmetry reduces near the defects, and the transverse components of electric field and induction as well as nonhomogeneous mechanical stresses must be taken into account. But the consideration of non-homogeneous mechanical stresses significantly complicates the problem, we hope that the system behavior would not change qualitatively under the influence of non-homogeneous mechanical stresses. The sample is regarded as linear dielectric in transverse $x, y$-directions and as nonlinear polar material in longitudinal $z$-direction.

\section{General equations}

Maxwell's equations for the electric induction D, field $\mathrm{E}$ and equation of continuity have the form:

$\operatorname{div} \mathrm{D}=4 \pi \rho, \quad \operatorname{rot} \mathrm{E}=0, \quad \frac{\partial \rho}{\partial t}+\operatorname{div} \mathrm{j}=0$.

They have to be supplemented by the equations of state:

$$
\begin{aligned}
& \mathrm{D}_{\perp}=\varepsilon_{\perp} \mathrm{E}_{\perp}, \quad D_{z}=D_{s}+\varepsilon_{z}(D) E_{z} \\
& \mathrm{j}=\sum_{m}\left(\mu_{m} \rho_{m} \mathrm{E}-\kappa_{m} \operatorname{grad} \rho_{m}\right), \quad \rho=\sum_{m} \rho_{m}+\rho_{s} .
\end{aligned}
$$

Here $\varepsilon_{z}$ and $\varepsilon_{\perp}$ are the longitudinal and transversal components of dielectric permittivity, $\rho_{m}, \mu_{m}$ and $\kappa_{m}$ are the $m$-th kind movable charge volume density, mobility and diffusion coefficient respectively, $\rho_{s}(\mathbf{r})$ is charge volume density of static defects. Also we suppose the transversal components of dielectric permittivity independent on the electric induction, i.e. the sample is treated as linear dielectric in this direction, but induction nonlinearly depends on the electric field in the longitudinal direction.

Dynamical equation of state for the induction $z$-component $D_{z} \equiv D$ can be obtained from the minimum of the Gibbs potential $G(D)$ with respect to the LandauKhalatnikov mechanism:

$$
\begin{aligned}
& \frac{\partial_{V}}{\partial_{V} D} G(D)=-\Gamma \frac{\partial D}{\partial t}, \\
& G(D)=\int_{V}\left(\frac{\alpha}{2} D^{2}+\frac{\beta}{4} D^{4}+\frac{\gamma}{2}\left(\frac{\partial D}{\partial \mathrm{r}}\right)^{2}-\mathrm{DE}\right) d \mathrm{r} .
\end{aligned}
$$

Here $\Gamma>0$ is the kinetic coefficient, $\alpha=\alpha_{T}\left(T-T^{*}\right), T$ is the absolute temperature, $T^{*}$ is the Curie temperature of the hypothetical pure (free of defects) sample, $\beta>0, \gamma>0$. Hereinafter symbol $\partial_{V}$ designates the variational derivative.

Note that the term DE is equal to the energy of free carriers $W=\int_{V} \rho \varphi d \mathrm{r}$. The Landau-Ginzburg-Devonshire equation determining the dependence of the induction on the electric field is follows from (3a), (3b).

$$
\Gamma \frac{\partial D}{\partial t}+\alpha D+\beta D^{3}-\gamma \frac{\partial^{2} D}{\partial \mathrm{r}^{2}}=E_{z}+\frac{\partial_{V}}{\partial_{V} D} \int_{V} \varepsilon_{\perp} E_{\perp}^{2} d \mathrm{r} .
$$




\section{A.N. Morozovska et al.: Dielectric response of disordered ferroelectrics with ...}

Equations (1), (2) can be rewritten as:

$$
\begin{aligned}
& \frac{\partial}{\partial z} D=4 \pi \rho-\varepsilon_{\perp} d i v_{\perp} \mathrm{E}_{\perp}, \\
& \operatorname{div}\left[\sum_{m=e, h}\left(\mu_{m} \rho_{m} \mathrm{E}-\kappa_{m} \operatorname{grad} \rho_{m}\right)+\right. \\
& \left.+\frac{1}{4 \pi} \frac{\partial}{\partial t}\left(D \mathrm{e}_{z}+\varepsilon_{\perp} \mathrm{E}_{\perp}\right)\right]=0 .
\end{aligned}
$$

Here $\mathbf{e}_{z}$ is the unit vector directed along $z$-axis.

Hereafter we suppose that the sample occupies the region $-\ell<z<\ell$ and homogeneous external field $E_{0}(t)$ is applied along $z$-axis. Boundary conditions depend on the mechanism of the spontaneous induction screening. If there is the thin non-ferroelectric layer with permittivity $\varepsilon_{e}$ the boundary conditions acquire the form:

$$
D(|z|=\ell) \approx \varepsilon_{e} E_{0}(t), \mathrm{E}_{\perp}(|z|=\ell)=0, \rho(|z|=\ell) \approx 0 .
$$

The latter condition from (4b) associates with the formation of oppositely charged space-charge layers with thickness $\ell_{c}$ near the non-ferroelectric layer (e.g. the Shottky barrier [15]). Let us consider that the applied field potential $\varphi$ is independent on transverse coordinates and is constant at $z= \pm \ell$. The sample is infinite and external field is absent in the transverse directions. So the inner field satisfies the conditions:

$$
\begin{aligned}
& \frac{1}{2 \ell} \int_{-\ell}^{\ell} E_{z}(\mathrm{r}, t) d z=\frac{\varphi(z=-\ell)-\varphi(z=\ell)}{2 \ell}=E_{0}(t), \\
& \int \mathrm{E}_{\perp}(\mathrm{r}, t) d \mathrm{r}_{\perp}=0 .
\end{aligned}
$$

Also we suppose that:

$$
\begin{aligned}
& f(\mathrm{r}, t)=\bar{f}(t)+\delta f(\mathrm{r}, t), \\
& \bar{f}(t)=\frac{1}{V} \int_{V} f(\mathrm{r}, t) d \mathrm{r} \Rightarrow \overline{\delta f(\mathrm{r}, t)}=0 .
\end{aligned}
$$

Hereinafter $f=\{\rho, E, D, j, \ldots\}$ and the dash designates the averaging over sample volume $V$.

It follows from (5a), (4c) that:

$$
E_{z}(\mathrm{r}, t)=E_{0}(t)+\delta E_{z}(\mathrm{r}, t), \quad \mathrm{E}_{\perp}(\mathrm{r}, t)=\delta \mathrm{E}_{\perp}(\mathrm{r}, t),
$$

i.e. $\bar{E}$ is the applied uniform field $E_{0}(t)$. Notice that the average values $\bar{E}, \bar{D}$ are determined experimentally [15], [16] most of the times. All the functions consist of the regular part caused by screening and the random one caused by fluctuations. Really, within the framework of our model one can write:

$$
\begin{aligned}
& \rho=\bar{\rho}(t)+\delta \rho(\mathrm{r}, t), \quad \rho_{s}(\mathrm{r})=\bar{\rho}_{s}+\delta \rho_{s}(\mathrm{r}), \\
& \delta \rho(\mathrm{r}, t)=\sum_{m=e, h} \delta \rho_{m}(\mathrm{r}, t)+\delta \rho_{s}(\mathrm{r}) .
\end{aligned}
$$

Here $\delta \rho_{m}(\mathrm{r}, t)$ is movable space-charge density of m-th kind, $\delta \rho_{s}(\mathrm{r})$ is the fluctuation of random static defects space-charge density, $\bar{\rho}_{s}$ is the average space-charge density of these defects. Further we limit ourselves by considering the bulk sample $\left(\ell>>\ell_{c}>>r_{c}\right)$ with quasihomogeneous distribution of $\delta \rho_{s}(\mathrm{r})$. In this case averaging (5a) over the sample volume is equivalent to the statistical averaging. Then as it follows from (5), (6) that auto-correlation function of the statical fluctuations $\rho_{s}^{2}(\mathrm{r})$ is the positive constant independent on time. Moreover, since the contribution to the integrals of the functions $\delta f^{n}(\mathrm{r}, t)$ from screening region is negligibly small in the bulk sample, and $d f$ are the fast oscillating functions in the remainder of the sample, one can conclude that:

$$
\begin{aligned}
& \overline{\delta f^{2 n+1}(\mathrm{r}, t)} \approx 0, \quad \overline{\delta f^{2 n}(\mathrm{r}, t)} \sim\left(\overline{\delta f^{2}(\mathrm{r}, t)}\right)^{n}, \\
& n=1 ; 2 \ldots f=\{\rho, E, D\} .
\end{aligned}
$$

Also we suppose that the correlation of the functions $\delta f$ product is equal to zero if the total power of different $\delta f$ is an odd number.

Having substituted (5) in (4a) and averaged, one can obtain the equations for the average quantities. Then "adding and subtracting" in (4a) nonzero correlation functions it is easy to obtain the equation for the fluctuations correlations compatible with the equation for the average values. The obtained self-consistent system provides the strong fulfillment of (4) and has the view:

$$
\begin{aligned}
& \bar{\rho}=0 \Rightarrow \sum_{m=e, h} \bar{\rho}_{m}=-\bar{\rho}_{s}, \quad \rho_{m}=\bar{\rho}_{m}+\delta \rho_{m}, \\
& \overline{\mathrm{j}}(t)=\overline{\mathrm{j}}_{c}(t)+\mathrm{e}_{z} \frac{\partial}{\partial t} \frac{\bar{D}(t)}{4 \pi}, \\
& \overline{\mathrm{j}}_{c}(t)=\sum_{m} \mu_{m}\left(\overline{\delta \mathrm{E} \delta \rho_{m}}+\mathrm{e}_{z} E_{0}(t) \overline{\rho_{m}}\right) .
\end{aligned}
$$

Here $\overline{\mathrm{j}}_{c}(t)$ is the macroscopic conductivity current. The absence of the space charge average density $\bar{\rho}$ follows from the sample electro-neutrality condition and corresponds to the result [15]. Using (5), (7a) one can obtain:

$$
\begin{aligned}
& \frac{\partial}{\partial z} \delta D=4 \pi\left(\sum_{m=e, h} \delta \rho_{m}+\delta \rho_{s}(\mathrm{r})\right)-\varepsilon_{\perp} d i v_{\perp} \delta \mathrm{E}_{\perp}, \\
& \operatorname{div}\left[\sum _ { m = e , h } \left(\mu_{m}\left(\delta \rho_{m} E_{0} \mathrm{e}_{z}+\left(\bar{\rho}_{m}+\delta \rho_{m}\right) \delta \mathrm{E}\right)-\right.\right. \\
& \left.\left.-\kappa_{m} \operatorname{grad} \delta \rho_{m}\right)+\frac{1}{4 \pi} \frac{\partial}{\partial t}\left(\mathrm{e}_{z} \delta D+\varepsilon_{\perp} \delta \mathrm{E}_{\perp}\right)\right]=0 .
\end{aligned}
$$

Using (7b), the quantities $\delta \rho_{m}, \delta \mathrm{E}$ can be expressed via the fluctuations of induction $\delta D$. Therefore using the relationship $\frac{\partial}{\partial D} \delta E_{\perp}^{2} \approx \frac{\partial}{\partial \delta D} \delta E_{\perp}^{2}$ and the nonlinear LGD equation (3b) it is easy to obtain self-consistent system: 


\section{A.N. Morozovska et al.: Dielectric response of disordered ferroelectrics with ...}

$$
\begin{aligned}
& \Gamma \frac{\partial \bar{D}}{\partial t}+\left(\alpha+3 \beta \overline{\delta D^{2}}\right) \bar{D}+\beta \bar{D}^{3}=E_{0}(t)+ \\
& +\varepsilon_{\perp} \frac{\partial_{V}}{\partial_{V} \delta D} \int_{V} \delta E_{\perp}^{2} d \mathrm{r}, \\
& \Gamma \frac{\partial}{\partial t} \delta D+\left(\alpha+3 \beta \bar{D}^{2}\right) \delta D+3 \beta \bar{D}\left(\delta D^{2}-\overline{\delta D^{2}}\right)+ \\
& +\beta \delta D^{3}-\gamma \frac{\partial^{2}}{\partial \mathrm{r}^{2}} \delta D= \\
& =\delta E_{z}+\varepsilon_{\perp}\left(\frac{\partial_{V}}{\partial_{V} \delta D} \int_{V} \delta E_{\perp}^{2} d \mathrm{r}-\frac{\partial_{V}}{\partial_{V} \delta D} \int_{V} \delta E_{\perp}^{2} d \mathrm{r}\right) .
\end{aligned}
$$

The system of equations (7) is closed. It determines the spatial-temporal evolution of the sample. In the case of bulk sample (7) has to be supplemented by the initial distributions of all variables.

Equation $(7 \mathrm{c})$ is valid for the bulk materials and represents itself the LGD-equation (3) for average (measurable) induction $\bar{D}$ with coefficient a renormalized by fluctuations (compare with modified susceptibility from [13] $\chi \sim 1 / \alpha_{R}$ :

$\alpha_{R}=\alpha+3 \beta \overline{\delta D^{2}}(t)$.

\section{Modified LGD-equation}

Unfortunately we failed to find the exact solution of nonlinear system (7b) and therefore to obtain the general form of $\delta D$ from (7d). However in the case of rather high movable charge concentrationm $\rho_{0}$ their mobility $\mu$ and smoothly varying applied field $E_{0}$ the characteristic time of $\delta \rho_{m}, \delta \mathbf{E}$ and $\delta D$ changing is the same order as maxwellian time $\tau_{m} \sim 1 / 4 \pi \mu \rho_{0}$ which is much smaller than $E_{0}$ period as well as Landau-Halatnikov relaxation time $\Gamma / \alpha$. Thus (7b) can be solved in the adiabatic approximation, i.e. after neglecting the temporal derivatives of $\delta \mathbf{E}$ and $\delta D$ compare with [17]). So one can obtain from $(7 a)$ and $(7 b)$ that:

$$
\sum_{m}\left(\mu_{m} \rho_{m}\left(\delta \mathrm{E}+\mathrm{e}_{z} E_{0}\right)-\kappa_{m} \operatorname{grad} \delta \rho_{m}\right)=\overline{\mathrm{j}}_{c}(t) .
$$

It follows from the definition (5a) $-\overline{\delta \mathrm{E}}=0$ and boundary conditions $(4 \mathrm{~b})$ that:

$$
\overline{\mathbf{j}}_{c}(t)=\mathbf{e}_{z} E_{0}\left(1 / \sum_{m} \mu_{m} \rho_{m}\right)^{-1} .
$$

Solving (9) as the transfer equation for the improper conductor with developed $n$-type conductivity. In order to avoid uncertainty:

$\sum_{m=e, h} \rho_{m}=-\bar{\rho}_{s}+\delta n, \quad \overline{\delta n}=0, \quad \mu<0, \quad \bar{\rho}_{s}>0$.

$S Q O, 6(2), 2003$

$$
\begin{aligned}
& \delta \mathrm{E} \approx \mathrm{e}_{z} E_{0}(t)\left(-1+\frac{\overline{1 /\left(\delta n-\bar{\rho}_{s}\right)}-1}{\delta n-\bar{\rho}_{s}}\right)+\frac{\kappa}{\mu} \operatorname{grad}\left(\ln \left|1-\frac{\delta n}{\bar{\rho}_{s}}\right|\right), \\
& \frac{\partial}{\partial z} \delta D \approx 4 \pi \bar{\rho}_{s}\left(\frac{\delta \rho_{s}}{\bar{\rho}_{s}}+\frac{\delta n}{\bar{\rho}_{s}}\right)-\varepsilon_{\perp} \frac{\kappa}{\mu} \Delta_{\perp}\left(\ln \left|1-\frac{\delta n}{\bar{\rho}_{s}}\right|\right)
\end{aligned}
$$

The equation (10c) with respect to $\delta n$ can be linearized and then solved in the quasi-neutral region of the sample, where:

$\left|\delta n / \bar{\rho}_{s}\right|<<1$,

$\delta \mathrm{E} \approx \mathrm{e}_{z} E_{0}(t) \frac{\delta n}{\bar{\rho}_{s}}-\frac{\kappa}{\mu \bar{\rho}_{s}} \operatorname{grad}(\delta n)$.

This solution has the form [18]:

$$
\begin{aligned}
& \delta n\left(\mathrm{r}_{\perp}, z, t\right)=\frac{1}{\lambda_{\perp}^{2}} \int_{S} d \widetilde{\mathrm{r}}_{\perp} K_{0}\left(\mathrm{r}_{\perp}-\widetilde{\mathrm{r}}_{\perp} \mid / \lambda_{\perp}\right) \times \\
& \times\left(\frac{\partial}{\partial z} \frac{\delta D\left(\widetilde{\mathrm{r}}_{\perp}, z, t\right)}{4 \pi}-\delta \rho_{s}\left(\tilde{\mathrm{r}}_{\perp}, z\right)\right) .
\end{aligned}
$$

Here $\mathrm{r}_{\perp}=(x, y), \lambda_{\perp}=\sqrt{-\frac{\varepsilon_{\perp} \kappa}{4 \pi \mu \bar{\rho}_{s}}}$ is the "transversal" screening length, $S$ is the sample cross-section, $K_{0}(r)$ is the Macdonald function. Having used solution (11b) and substituted (10b) into (7d), we obtain nonlinear integraldifferential equation for $\delta D$ with proportional to $\delta \rho_{s}$ non-homogeneity. Assuming that movable free-carriers can screen at least partially the charged clusters $\delta \rho_{s}$, one can conclude from (11) and (7b), (7d) that:

$$
\begin{aligned}
& \frac{\delta D}{4 \pi} \sim \lambda_{z} \hat{\eta}\left(\delta \rho_{s}\right), \quad \frac{\partial}{\partial z} \frac{\delta D}{4 \pi} \sim \hat{\eta}\left(\delta \rho_{s}\right), \\
& \delta n \sim \widehat{L}\left[\frac{\partial}{\partial z} \frac{\delta D}{4 \pi}-\delta \rho_{s}\right], \quad \hat{\eta}[0]=0
\end{aligned}
$$

Here $\lambda_{z}$ is characteristic "longitudinal" screening length, $\hat{\eta}$ is unknown nonlinear odd at $\delta \rho_{s}$ operator-function, which depends on time, applied field, temperature, coefficients $\alpha, \beta, \gamma$ and other material constants, and $\bar{L}$ is operator linear under approximation (11a). In order to obtain the approximate expressions for unknown in (7c) variables, let us make some simplifications. Using representation (12) and solution (11) one estimates the necessary correlation functions.

Using (10b), (11) and (6b) it is easy to derive that:

$$
\frac{\partial_{V}}{\partial_{V} \delta D} \int_{V} \delta E_{\perp}^{2} d \mathrm{r} \approx 0 .
$$

After integrating over parts allowing for (4)-(5) one can obtain: 


$$
\begin{aligned}
& \overline{\delta D \frac{\partial^{2}}{\partial \mathrm{r}^{2}} \delta D}=\overline{\left(\frac{\partial}{\partial \mathrm{r}} \delta D\right)^{2}} \sim R_{1}\left(\overline{\delta \rho_{s}^{2}}\right) \\
& \overline{\delta D \frac{\partial}{\partial z} \delta n}=-\overline{\delta n \frac{\partial}{\partial z} \delta D} \sim R_{2}\left(\overline{\delta \rho_{s}^{2}}\right) \\
& \overline{\delta D \delta n} \sim \lambda_{z} R_{3}\left(\overline{\delta \rho_{s}^{2}}\right) \\
& \delta D \frac{\partial_{V}}{\partial_{V} \delta D} \int_{V} \delta E_{\perp}^{2} d \mathrm{r}=0
\end{aligned}
$$

Here $b$ is average transverse size of charged clusters. Functions $R_{n}$ depend on time, applied field $E_{0}(t)$, temperature $T$ and other material constants. Note, that $R_{n}(0)=0$ and their absolute values increase with $\delta \rho_{s}^{2}$ increasing. One can estimate from (11a) that:

$$
\overline{\delta E_{\perp}^{2}} / \overline{\delta E_{z}^{2}} \sim \lambda_{\perp}^{2} / b^{2}
$$

Actually (16) means, that transverse components of electric fields can be neglected after the averaging of equations, only if the average transverse size $b$ of charged clusters is much greater than the transverse screening length $\lambda_{\perp}$.

The equation for average induction $\bar{D}(t)$ can be derived from (13) and (7c) as following:

$$
\Gamma \frac{\partial \bar{D}}{\partial t}+\left(\alpha+3 \beta \overline{\delta D^{2}}\right) \bar{D}+\beta \bar{D}^{3}=E_{0}(t)
$$

Multiplying (7d) on $\delta D$ and averaging, the equation for the average square of the induction fluctuation $\overline{\delta D^{2}}(t)$ can be derived in the form:

$$
\begin{aligned}
& \frac{\Gamma}{2} \frac{\partial \overline{\delta t} \overline{\delta D^{2}}+\left(\alpha+3 \beta \bar{D}^{2}(t)\right) \overline{\delta D^{2}}+\beta\left(\overline{\delta D^{2}}\right)^{2}=}{=} \overline{\delta D \frac{\partial^{2}}{\partial \mathrm{r}^{2}} \delta D}+{\overline{\delta D \delta E_{z}}}_{z}
\end{aligned}
$$

The right-hand side of the equation (17b) within the framework of our approximations and estimations has the form:

$$
-\gamma \overline{\left(\frac{\partial}{\partial z} \delta D\right)^{2}}+\overline{\delta D \delta E}_{z}=R_{S}\left(\overline{\delta \rho_{s}^{2}}\right)
$$

Function $R_{S}$ can be estimated from (11a), (12), (14)-(15) in the terms of the functions $R_{n}$ it depends on time, external field $E_{0}(t)$ frequency and amplitude, temperature $T$ etc. It can be shown from the general relationship (7b) and (10), that $R_{S}(0)=0$ and its modulus increases with $\delta \rho_{s}^{2}$ increase. It is evident, because $R_{s}$ is the source of the induction fluctuations in (17b) and these fluctuations increase with the charge density fluctuation $\delta \rho_{s}^{2}$ increasing. The magnitude $R_{s}$ is negative in the absence of the electric field fluctuations $\delta \mathrm{E}$ and increases with transverse field $\delta E_{\perp}^{2}$ increasing. In the case of rather significant field fluctuations $R_{S}$ becomes positive.

Equations (17)-(18) compose the self-consistent system. We would like to emphasize that in deriving the equation (17a) we use only the formula (13), but do not use the approximate estimations (14)-(15). That is why the region where $(17 \mathrm{a})$ is valid is much wider than the one for expression (18), which determines right-hand side of the equation (17b).

Equation (17a) represents itself the modified LGDequation for bulk samples with developed induction fluctuations (see [13] for comparison).

The system (17) has the following physical interpretation. The state of the sample with charged defects can be described by two order parameters. The long-range order parameter $\bar{D}$ describes the ferroelectric ordering in the system, and the local order parameter $\overline{\delta D^{2}}$ describes disordering caused by strong electric fields (in comparison with matrix coercive field) arising near charged non-homogeneities $\overline{\delta \rho_{s}^{2}}$. We will show that the zero-field (i.e. $E_{0}(t) \equiv 0$ phase transition into the state $\bar{D}=0, \overline{\delta D^{2}} \neq 0$ takes place at the critical concentration of non-homogeneities $\overline{\delta \rho_{s}^{2}}$ i.e. the sample splits into the polar regions with opposite induction orientation (actually Känzig regions), and the system behaves as DF.

\section{Dielectric hysteresis and permittivity}

1) Let us underline the following properties of modified LGD-equation (17a).

The fact that the known applied external field $E_{0}$ stands in the right hand-side of (17a), is its main advantage in comparison with (3b), where the unknown field $\left.E=E_{0}(t)+\delta E(\mathbf{r}, t) 1\right)$ stands in the right hand-side.

2) Under obtaining equation (17a) we did not assume the smallness of induction fluctuations $\delta D$ This means, that our results can describe the DF-materials adequately, where the induction fluctuations are the same order or more that its mean value. The modified LGD-equation (17a) admits the continuous transformation from the ordered LGD ferroelectric $\left(\overline{\delta D^{2}}(t)<<|\alpha| / 3 \beta\right)$ to the $\left(\overline{\delta D^{2}}(t) \leq|\alpha| / 3 \beta\right)$ and then to the completely disordered material $\left(\overline{\delta D^{2}}(t)>|\alpha| / 3 \beta\right)$ under increasing $\overline{\delta D^{2}}(t)$.

Notice, that $\left|\alpha_{R}\right|<|\alpha|$ and $\alpha_{R}$ depends on time, thus the dielectric hysteresis loop $\bar{D}\left(E_{0}\right)$ becomes $\left(|\alpha| /\left|\alpha_{R}\right|\right)^{3 / 2}$ times narrower and $\left(|\alpha| /\left|\alpha_{R}\right|\right)^{1 / 2}$ times lower, also it could change its shape (e.g. transforms into the double one) and acquire the non-stationary character (see for details Fig. 1-4). 


\section{A.N. Morozovska et al.: Dielectric response of disordered ferroelectrics with ...}

Let us rewrite equations (17) allowing for (18) in dimensionless variables.

$$
\frac{\partial}{\partial \tau} D_{m}(\tau)+(\operatorname{sign}(\alpha)+3 \Delta(\tau)) D_{m}(\tau)+D_{m}^{3}(t)=E_{m}(\tau),
$$

$\frac{\partial}{\partial \tau} \frac{\Delta(\tau)}{2}+\left(\operatorname{sign}(\alpha)+3 D_{m}^{2}(\tau)\right) \Delta(\tau)+\Delta^{2}(\tau)=R$,

$D_{m}(0)=D_{m 0}, \quad \Delta(0)=\Delta_{0}$.

Here $D_{m}(\tau)=\bar{D}(t) / D_{s}, \Delta(\tau)=\overline{\delta D^{2}}(t) / D_{s}^{2}, E_{m}(\tau)=$ $E_{0}(t) /\left(|\alpha| D_{s}\right), R(T)=R_{s} /\left(\alpha \mid D_{s}^{2}\right), D_{s}(T)=\sqrt{\alpha(T) \mid / \beta}$,
$\alpha(T)=\alpha_{T}\left(T-T^{*}\right), \operatorname{sign}(0)=1$. In the case of periodic applied field $E_{m}(\tau)=E_{m 0} \sin (w \tau), \quad w=\omega \Gamma /|\alpha|$.

Let us study the stability of system's (19) zero-field stationary solutions. In the case function $R$ can be regarded time-independent constant. The stability of the stationary points can be determined from the behavior of small deviations from the ones. Obviously that the existence and stability of stationary solutions (19) depend on parameter $R$ values. The frequency dispersion of dielectric permittivity $\varepsilon(\omega)$ can be found after linearizing of (19) near the stationary points for the small periodic applied field. The stability of stationary points $\left(D_{s}\right.$ and $\left.D_{m s}\right)$, dispersion of dielectric permittivity $\varepsilon(\omega)$ and the shape
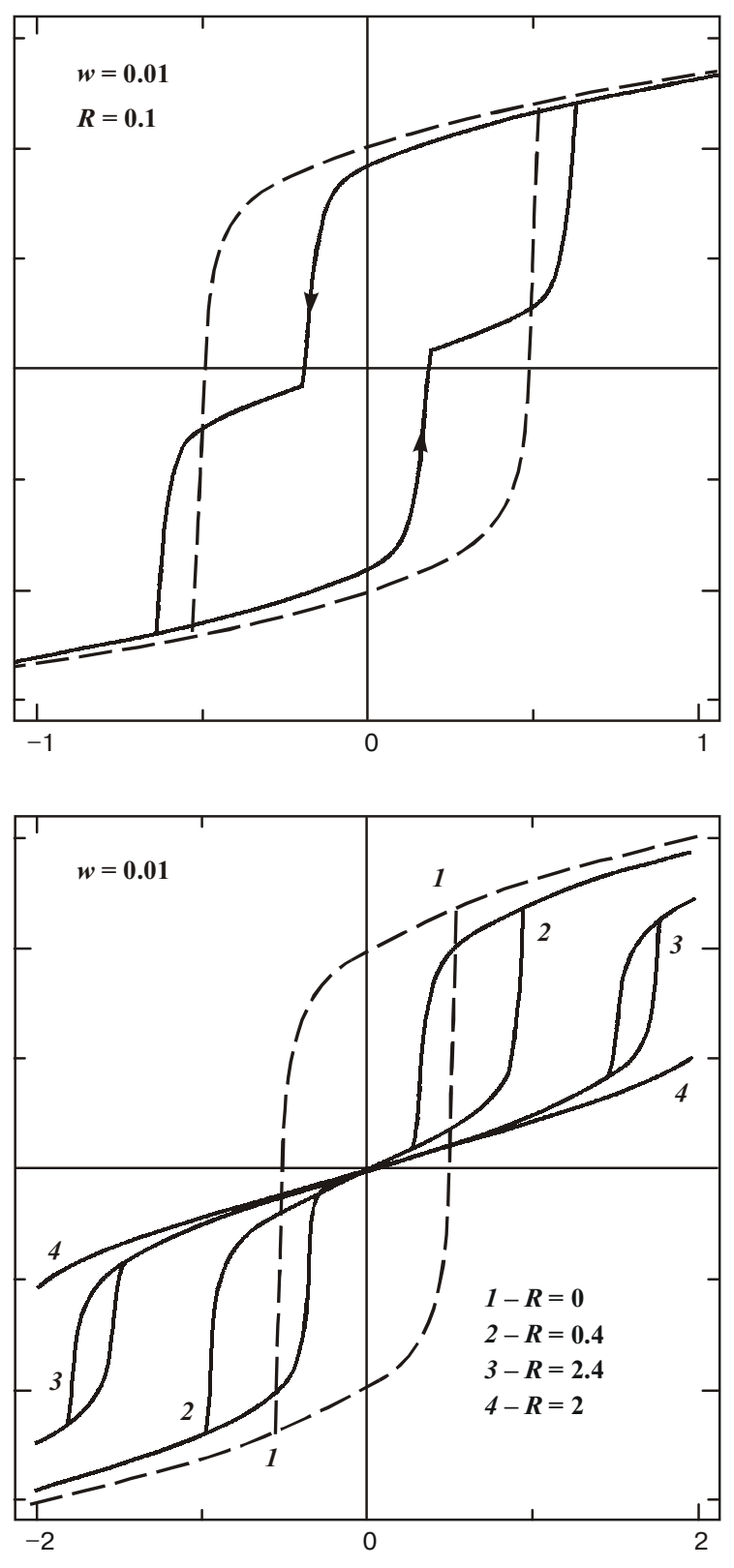

$\boldsymbol{E}_{m}$

Fig. 1. The quasi-static dependence of dimensionless induction on dimensionless field (hysteresis loops) for constant frequency $w=0.01$ and temperature at different $R$ values marked by figures near solid curves. The dashed curve corresponds to the "classic" loop in the ordered ferroelectrics $(R=0)$ at the same frequency value. 


\section{A.N. Morozovska et al.: Dielectric response of disordered ferroelectrics with ...}

of hysteresis loops $D_{m}\left(E_{m}\right)$ for different intervals of parameter $R$ are discussed below. For hysteresis loops investigation we suppose that $\alpha<0$ and $R$ is independent both on time and applied electric field.

1. $\alpha>0, R<0$. Stationary states at $\Delta_{S}>0$ are absent.

2. $\alpha>0, R>0$. One steady stationary state $D_{m s}=0$, $\Delta_{s}=\frac{-1+\sqrt{1+4 R}}{2}$ is present. The Debye dispersion law is valid: $\varepsilon(\omega)=\frac{1}{|\alpha(T)|\left(3 \Delta_{s}+1\right)-i \Gamma \omega}$.
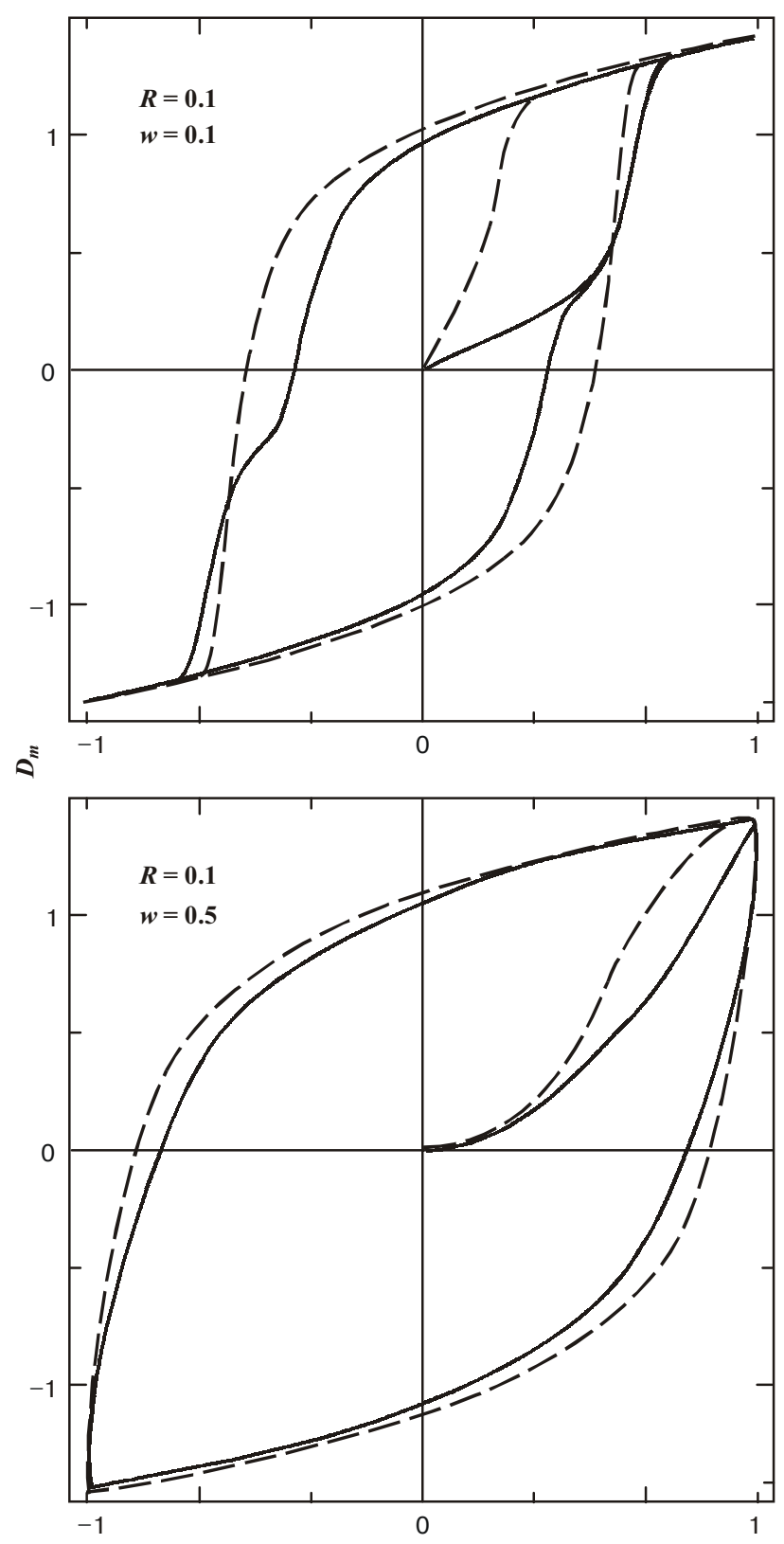

3. $\alpha<0,-\frac{1}{4}<R<0$. Stationary states at $\Delta_{s}>0$ are absent. This is the region of determined chaos.

4. $\alpha<0,-\frac{1}{4}<R<0$. The steady state $D_{m s}=0$, $\Delta_{s}=\frac{1+\sqrt{1+4 R}}{2}$ corresponds to the local minimum of $G(D)$ nonphysical steady states at $\Delta_{S}<0$ correspond to the global minimum of $G(D)$. Because of this fact the static hysteresis loops (i.e. obtained at infinitely small rate of the applied field changing) at $\Delta_{s}>0$ are absent.
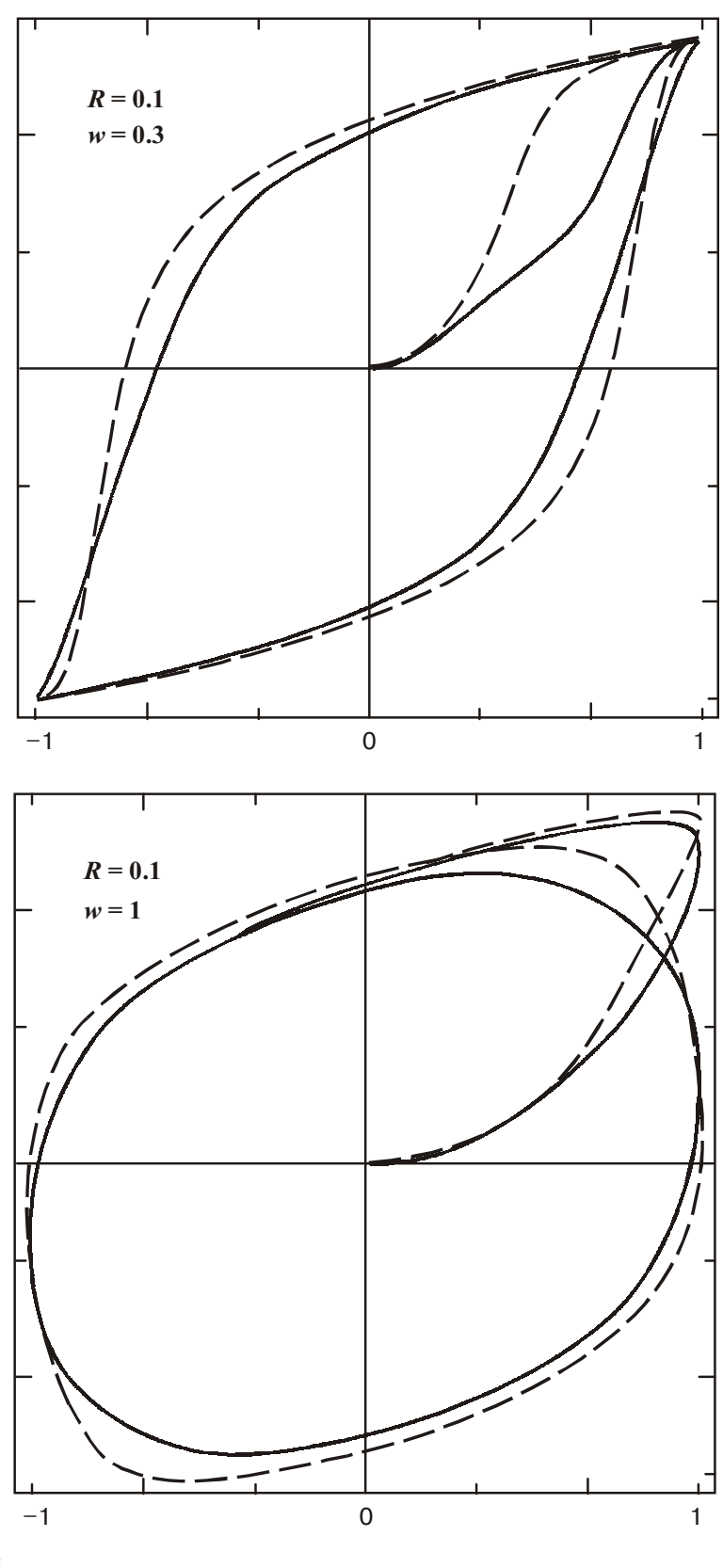

Fig. 2. The quasi-static dependence of dimensionless induction on dimensionless field (hysteresis loops) for the value $R=0.1$ and temperature at different frequency w values marked by figures near solid curves. The dashed curve corresponds to the "classic" loop in the ordered ferroelectric $(R=0)$ at the same frequency value. 


\section{A.N. Morozovska et al.: Dielectric response of disordered ferroelectrics with ...}
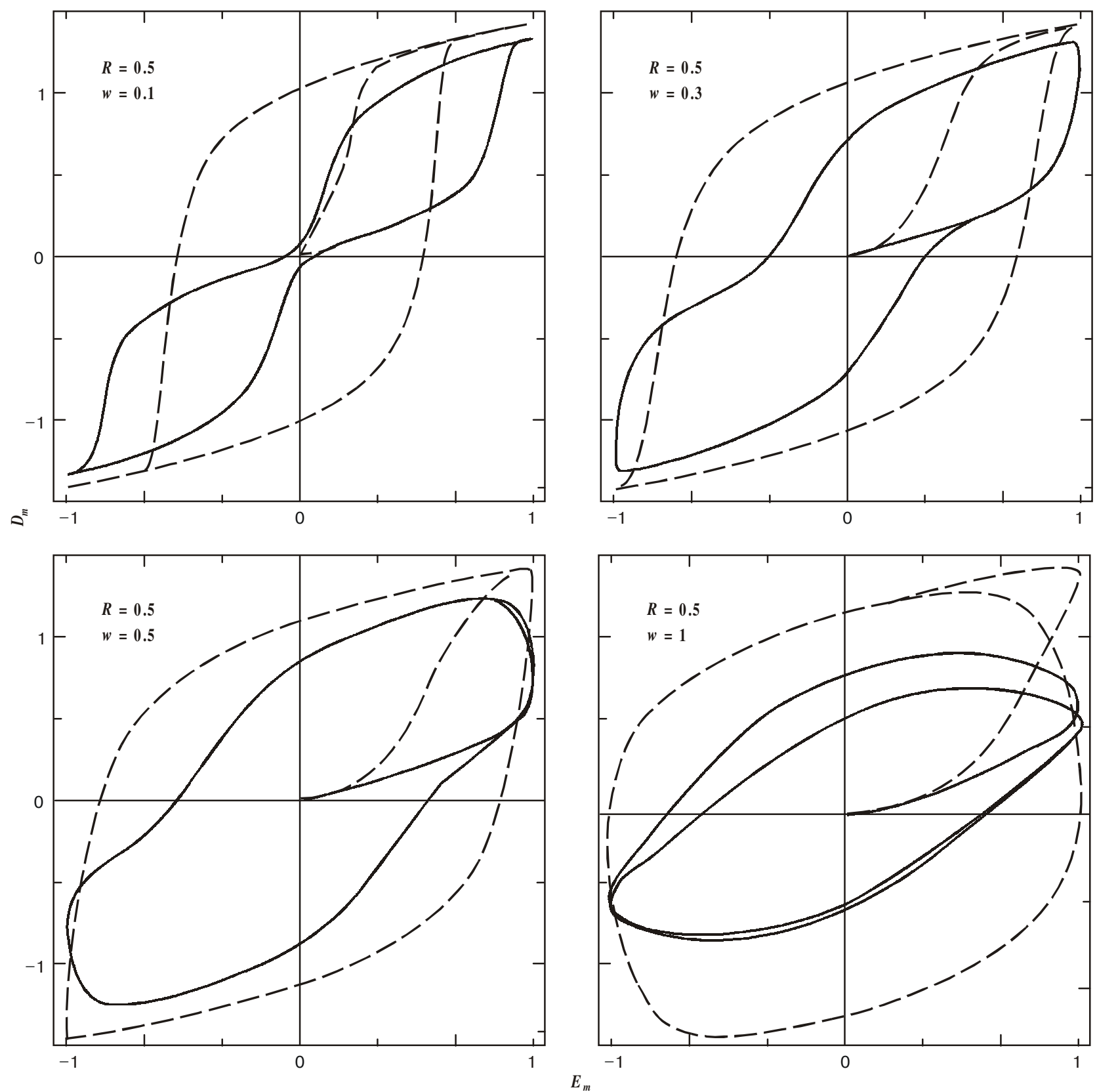

Fig. 3. The quasi-static dependence of dimensionless induction on dimensionless field (hysteresis loops) for the value $R=0.5$ and temperature at different frequency w values marked by figures near solid curves. The dashed curve corresponds to the "classic" loop in the ordered ferroelectrics $(R=0)$ at the same frequency value.

5. $\alpha<0,0 \leq R<\frac{1}{8}$. Together with the steady state $D_{m s}=0$, $\Delta_{s}=\frac{1+\sqrt{1+4 R}}{2}$ there exists another one $D_{m s}^{2}=1-3 \Delta_{s}$, $\Delta_{s}=\frac{1-\sqrt{1-8 R}}{8}$. The latter state ordered partially at $R>0$ and completely at $R=0$ corresponds to the global minimum of $G(D)$ In this case dielectric response of the system $\varepsilon(\omega)$ reveals the non-Debye behaviour with additional dependence on the external field frequency and temperature:

$$
\begin{aligned}
& \varepsilon(\omega)= \\
& =\frac{2|\alpha(T)|\left(2-7 \Delta_{s}\right)-i \Gamma \omega}{8|\alpha(T)|^{2}\left(1-3 \Delta_{s}\right)\left(1-8 \Delta_{s}\right)-2 i|\alpha(T)|\left(3-10 \Delta_{s}\right) \Gamma \omega-\Gamma^{2} \omega^{2}}
\end{aligned}
$$

This is the region of the partially ordered ferroelectrics. The static hysteresis loops are similar to the "classic" one (i.e. $R=0$ at), but the refinement appears and becomes more narrow with $R$ increasing (see Fig. 1a, b). The refinement disappears with frequency increasing, and the loop shape almost coincides with the classical one (see Fig. 2). 


\section{A.N. Morozovska et al.: Dielectric response of disordered ferroelectrics with ...}

6. $\alpha<0, \frac{1}{8} \leq R$. One steady state $D_{m s}=0, \Delta_{s}=$ $\frac{1+\sqrt{1+4 R}}{2}$. The Debye dispersion Law is valid: $\varepsilon(\omega)=\frac{1}{|\alpha(T)|\left(3 \Delta_{s}-1\right)-i \Gamma \omega}$. This is the region of $D F$ consisted of polar regions with opposite polarization orientation. The static hysteresis loops are similar to the double "pseudo-antiferroelectric" [19] ones (see Fig. 1c, d). Double loops transforms into single ones under frequency increasing, but they are much narrower than the classical loop and acquires the relaxational character (see Fig. 3, 4).

The dependence of steady order parameters and static susceptibility over temperature (see Fig. 5) can be obtained from the aforementioned speculations, i.e.:

$$
\begin{aligned}
& \frac{\bar{D}(T)}{D_{s}(0)}=\theta\left(\frac{T}{T^{*}}-1-\sqrt{8 R(0)}\right) \times \\
& \times \sqrt{\frac{5}{8}\left(\frac{T}{T^{*}}-1\right)+\frac{3}{8} \sqrt{\left(\frac{T}{T^{*}}-1\right)^{2}-8 R(0)}}
\end{aligned}
$$
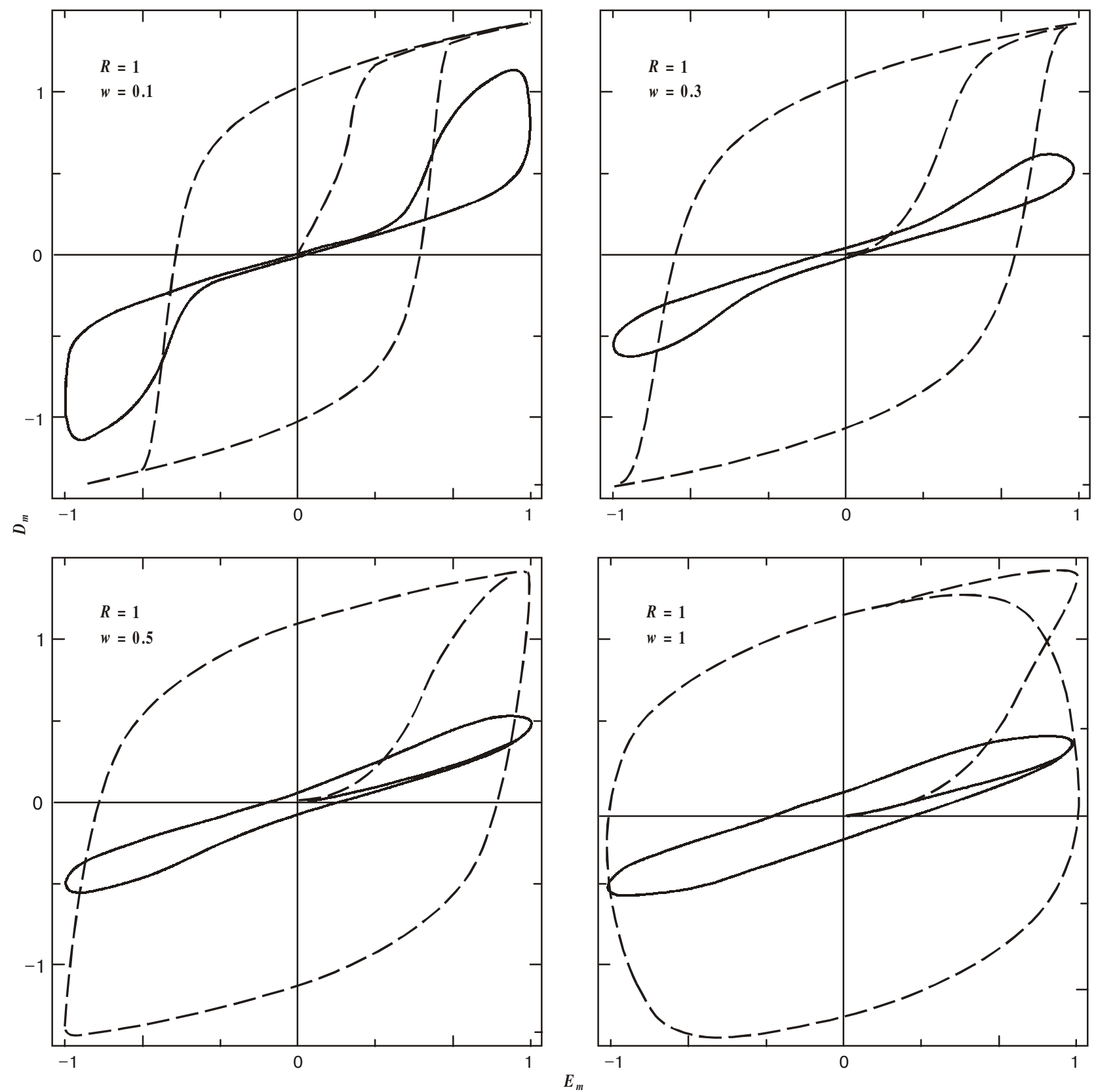

Fig. 4. The quasi-static dependence of dimensionless induction on dimensionless field (hysteresis loops) for the value $R=1$ and temperature at different frequency w values marked by figures near solid curves. The dashed curve corresponds to the "classic" loop in the ordered ferroelectrics $(R=0)$ at the same frequency value. 


$$
\begin{aligned}
& \frac{\sqrt{\frac{\delta D^{2}(T)}{D_{s}(0)}}=\theta\left(\frac{T}{T^{*}}-1-\sqrt{8 R(0)}\right) \times}{\times \sqrt{\frac{1}{8}\left(\frac{T}{T^{*}}-1\right)-\frac{1}{8} \sqrt{\left(\frac{T}{T^{*}}-1\right)^{2}-8 R(0)}+}} \\
& +\theta\left(\sqrt{8 R(0)}-\frac{T}{T^{*}}+1\right) \times \\
& \times \sqrt{\frac{1}{2}\left(\frac{T}{T^{*}}-1\right)+\frac{1}{2} \sqrt{\left(\frac{T}{T^{*}}-1\right)^{2}+4 R(0)}} .
\end{aligned}
$$

Here $\theta(x)$ is step-function. It is clear from the Fig. 5 that long-range order parameter $\bar{D}$ reduces when temperature increases and sharply becomes zero at some critical point determined by $R(T=0)$ i.e. by $R_{s}\left(\overline{\delta \rho_{s}^{2}}\right)$. The local order parameter $\sqrt{\delta D^{2}}$ monotonically increases when temperature increases up to the critical point, where in it sharply increases and then monotonically decreases and tends to zero at $T \rightarrow \infty$. The static susceptibility (see inset in the Fig. 5) diverges when approaching the critical point from low temperatures ("sharp" maximum), then it abruptly decreases to finite value and under farther temperature increasing demonstrates the "wide" maximum. Both maximums coincide at cal point from low temperatures ("sharp" maximum), then it abruptly de-

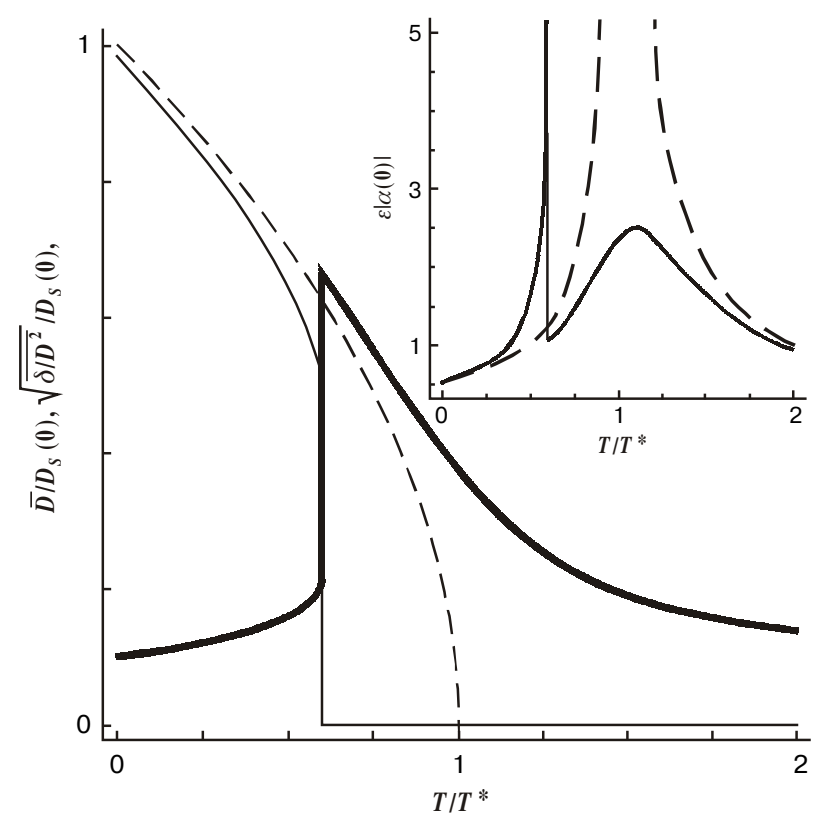

Fig. 5. The dependence of dimensionless spontaneous induction (thin solid curve) and local induction (thick solid curve) on temperature for the value $R(0)=0.02$. The dashed curve corresponds to the spontaneous induction of the ordered ferroelectrics $(R=0)$. The static permittivity dependence on temperature is depicted in the inset. creases to finite value and under farther temperature increasing demonstrates the "wide" maximum. Both maximums coincide at $R(0) \rightarrow 0$ and the distance between them is the longer the bigger is $R(0)$, until "sharp" maximum disappears at $R(0) \geq 1 / 8$.

Thus, the dielectric response of the system has anomalies not only when the long-range order parameter $\bar{D}$ originates, as in the ordered ferroelectrics, but also when the local order parameter $\sqrt{\overline{\delta D^{2}}}$ increases. Similar coexistence of the dielectric permittivity anomalies is observed by the authors of ref. [20] in the $\mathrm{Pb}_{0.9} \mathrm{La}_{0.1} \mathrm{TiO}_{3}$ ceramics. Position of the narrow low temperature peak of the permittivity is found independent on the frequency, but the high temperature one is essentially shifted with the external field frequency. This gives basis to authors of ref. [20] to connect the aforementioned anomalies with the phase transitions from ordered ferroelectric state to the relaxor state and from relaxor state to paraelectric phase respectively. Phase transition of the relaxor material to the ordered state (in the absence of the external polarizing field) is a intrinsic feature of many relaxor ferroelectrics (see e.g. [21] and ref. therein) and can manifest itself as an abrupt curve break of the permittivity dependence on temperature. The distinctive peculiarity of this anomaly is its position independence on the frequency.

\section{Dicussion}

1. Double pseudo-antiferroelectric loops [19] are observable in some cases in the ferroelectric materials. For example, "refined" loops arise as a result of the normal ferroelectric loops distortions by the internal charged nonhomogeneities, leading to the electric field fluctuations (e.g. $\mathrm{BaTiO}_{3}$ and $(\mathrm{Pb}, \mathrm{Ca}) \mathrm{TiO}_{3}$ ceramics). Also «refined» and pseudo-antiferroelectric hysteresis loops exist in the plumbum zirconate-titanate ceramics doped with $\mathrm{La}$ [1], namely in $\mathrm{Pb}_{1-\mathrm{y}} \mathrm{La}_{\mathrm{y}} \mathrm{Zr}_{\mathrm{x}} \mathrm{Ti}_{1-\mathrm{x}} \mathrm{O}_{3}$ at $x=0.35, y=0.08,0.084$ and $x=0.3, y=0.076,0.079$, which is regarded as relaxor material. Double loops can be observable in ferroelectric ceramics samples if applied field is perpendicular to the initial polarization direction (e.g. $\mathrm{Pb}(\mathrm{Ti}, \mathrm{Zr}) \mathrm{O}_{3}$ [19]), which can be explained as the pinning of domain walls by internal mechanical stresses and charged defects.

2. We would like to underline, that static hysteresis loops are theoretical abstraction. For real DF even the lowest possible frequency of the applied field can be much higher than the inverse eigen relaxation time of $\Gamma /|\alpha|$, and so it is too high for static loop observation. Let us remind that $\Gamma, \alpha$ are coefficients for hypothetical pure sample they can be treated as fitting parameters. Therefore, when applying our model to real hysteresis loops in relaxors, the narrow "dynamic" loops would be compared with the experiment rather than double «static» ones.

3. Notice, that at $0<R<\frac{1}{8}$ the static susceptibility of partly disordered ferroelectri: 


$$
\varepsilon(0)=\frac{\left(1-3.5 \Delta_{s}\right)}{2|\alpha(T)|\left(1-3 \Delta_{s}\right)\left(1-8 \Delta_{s}\right)}, \Delta_{s}=\frac{1-\sqrt{1-8 R}}{8}
$$

is higher than the one in the ordered sample $(R=0)$, and diverges at $R \rightarrow 1 / 8$ ("sharp" maximum). Within the framework of our model both the non-homogeneous concentration of charged defects $\left(\delta \rho_{s} \neq 0\right)$ and the improper conductivity caused by it $(\delta n \neq 0)$ do provide the aforementioned anomalies of dielectric response. The frequency dispersion of dielectric susceptibility temperature maximum can be derived if the dependence of $R$ over applied field is known. This problem is in progress now.

4. In the case when the mean transversal size of the charged non-homogeneities is comparable or less than the transverse screening length: $b \leq \lambda_{\perp}$, it is necessary to take into account the transverse components of electric field. In particular, only after taking into consideration all the components of electric fields caused by charged nano- and micro- inclusions, the critical fluctuations of their concentration $\overline{\delta \rho_{s}^{2}}$ and real sizes of polar regions arisen around them can be estimated correctly. The structure of these fields is much closer to the vanishing at great distances radial fields of points charges, which are able to repolarize only micro-regions, than to the non-vanishing uniform longitudinal fields of charged plains, which posses infinite energy and can repolarize macro-regions. Neglecting of the transverse fields could lead to the significantly over-estimated critical values of $\overline{\delta \rho_{s}^{2}}$, i.e. at $R<0$ ordered state is unstable.

5. We can conclude, that modified LGD-equation (17) describes adequately the disordering (namely polarization switching phenomena and some dielectric properties), caused by charged clusters in such bulk ferroelectric materials with improper conductivity as $\mathrm{BaTiO}_{3}$, $(\mathrm{Pb}, \mathrm{Ca}) \mathrm{TiO}_{3}$ ceramics and relaxor PLZT-ceramics.

\section{Acknowledgments}

The authors are greatly indebted to Prof. N.V. Morozovsky for frutfull discussions of the model and useful remarks to the manuscript. The work is supported by grant INTAS-01-0173.

\section{References}

1. Q.Y. Jiang, E.C. Subbarao and L.E. Cross, Effect of compositional and temperature on electric fatigue of Ladoped lead zirconate titanate ceramics // J. Appl. Phys. 75(11), pp.7433-7443 (1994).

2. G.A. Smolensky, V.A. Isupov, A.I. Agranovskaya, S.N. Popov, Ferroelectrics with diffuse phase transition // Fiz. Tverd. Tela 2(10), pp. 2906-2913 (1960).

3. B.N. Rolov, Influence of compositional fluctuations on the diffusion of the ferroelectric phase transitions // Fiz. Tverd. Tela 6(9), pp. 2128-2131 (1964) [Sov. Phys.-Solid State 6(9), pp.1676-1679 (1965)].

4. L.E. Cross, Relaxor ferroelectrics // Ferroelectrics, 76, pp. 241-245 (1987).

5. A.J. Bell, Calculation of dielectric properties from the superparaelectric model of relaxors // J. Phys.: Cond. Matter, 5, pp. 8773-8792 (1993).

6. D. Viehland and J.-F. Li, The role of local compositional instability in mixed B-site cation relaxors // Ferroelectrics 158, pp. 381-386 (1994).

7. A.E. Glazounov, A.J. Bell and A.K. Tagantsev, Relaxors as superparaelectrics with distributions of the local transition temperature // J. Phys.: Cond. Matter 7, pp. $4145-4168$ (1995).

8. Sh.Li, J.A.Eastman, R.E.Newnham and L.E.Cross, Diffuse phase transition in ferroelectrics with mesoscopic heterogeneity: Mean-field theory // Phys. Rev. B 55(18), pp.1206712078 (1997).

9. B.E.Vugmeister and H.Rabitz, Dynamics of interacting clusters and dielectric responce in relaxor ferroelectrics // Phys. Rev., B 57(13), pp.7581-7585 (1998).

10. B.E.Vugmeister and H.Rabitz, A phenomenology of relaxor ferroelectrics // Ferroelectrics 201, pp. 33-42 (1997).

11. M.D.Glinchuk and R.Farhi. A random field theory based model for the ferroelectric relaxors // J. Phys.: Cond. Matter, 8, pp. 6985-6996 (1996).

12. R.F. Mamin, About the theory of the phase transition in relaxors // Fiz. Tverd. Tela, 43(7), pp.1262-(2001)

13. B.Kirsh, H.Schmitt and H.Muser. // Ferroelectrics, 68, pp. 275 284 (1986).

14. D. Viehland, S.J Jang, L.E. Cross, M. Wuttig, Deviation from Curie-Weiss behavior in relaxor ferroelectrics // Phys. Rev., B 46(13), pp.8003-8006 (1992).

15. V.M. Fridkin, Ferroelectrics semiconductors, chap.3, Consultant Bureau, New-York and London (1980).

16. M.E. Lines and A.M.Glass, Principles and Applications of Ferroelectrics and Related Phenomena, Oxford University Press (1978).

17. G.M. Guro, I.I. Ivanchik, N.F. Kovtonuk, Semiconducting properties of barium titanate // Fiz. Tverd. Tela, 10(1) pp.135143 (1968) [Sov. Phys.-Solid State 11(1), pp.1956-1964 (1969)]

18. A.N. Tikhonov, A.A. Samarsky, Methods of the mathematical physics, pp.653-655, Nauka, Moscow (1972).

19. B. Jaffe, W.R.TCook and H. Jaffe, Piezoelectric Ceramics, Academic Press, London and New York (1971).

20. M. Kuwabara, K. Goda and M. Oshima, Coexistence of normal and diffuse ferroelectrics-paraelectric phase transitions in $(\mathrm{Pb}, \mathrm{La}) \mathrm{TiO}_{3}$ ceramics // Phys. Rev., B 42(16), pp.1001210015 (1990).

21. G.A. Samara, Pressure-induced crossover from long- to shortrange order in compositionally disordered soft mode ferroelectrics // Phys. Rev. Lett., 77(2), pp.314-317 (1996). 